\title{
Exenatide Once Weekly for Management of Type 2 Diabetes: A Review
}

\author{
Jun Inaishi' ${ }^{1,2}$, Yoshifumi Saisho $\mathbb{D}^{1}$ \\ 'Division of Endocrinology, Metabolism and Nephrology, Department of Internal Medicine, Keio University School of Medicine, Tokyo, Japan; ${ }^{2}$ Center for \\ Preventive Medicine, Keio University School of Medicine, Tokyo, Japan \\ Correspondence: Yoshifumi Saisho, Division of Endocrinology, Metabolism and Nephrology, Department of Internal Medicine, Keio University School \\ of Medicine, 35 Shinanomachi, Shinjuku-ku, Tokyo, 160-8582, Japan, Tel +8I-3-3353-12II (x62383), Fax +8I-3-3359-2745, Email ysaisho@keio.jp
}

\begin{abstract}
Exenatide is one of the exendin-based glucagon-like peptide 1 receptor agonists (GLP-1RAs) and is currently available in two formulations, ie, exenatide twice daily (BID), a short-acting GLP-1RA, and exenatide once weekly (QW), a long-acting GLP1RA. Clinical efficacy and safety of exenatide $2 \mathrm{mg}$ QW in patients with type 2 diabetes (T2DM) has been demonstrated in the DURATION study program. Exenatide QW has been shown to achieve greater HbA1c reduction compared with exenatide BID, with less injection frequency and greater treatment satisfaction. However, exenatide QW failed to show a significant cardiovascular risk reduction in a cardiovascular outcome trial (CVOT), the EXSCEL trial, while other GLP-1RAs have shown positive CV outcomes. Furthermore, exenatide QW has been shown to be inferior to liraglutide and semaglutide with respect to HbA1c or body weight reduction in the head-to-head trials. Thus, although the long-term efficacy and safety of exenatide QW have been demonstrated, exenatide QW might be selected with lower priority within the class of GLP1-RAs for the management of T2DM, especially for patients at high CV risk. On the other hand, exenatide QW is now expected to be a treatment option for children with T2DM or patients with Parkinson's disease. This review provides an overview of the current evidence regarding the clinical efficacy and safety of exenatide QW and discusses the current perspectives on exenatide QW for treatment of T2DM.
\end{abstract}

Keywords: exenatide once weekly, GLP-1 receptor agonist, type 2 diabetes, cardiovascular outcome

\section{Introduction}

In the past two decades, new anti-diabetic medications for diabetes have been developed and marketed, including incretin-based therapy, which consists of two different classes: dipeptidyl peptidase-4 (DPP-4) inhibitors and glucagonlike peptide-1 (GLP-1) receptor agonists (GLP-1 RAs). DPP-4 inhibitors enhance the plasma levels of native GLP-1 and glucose-dependent insulinotropic polypeptide by preventing their proteolytic degradations, while GLP-1 RAs display structural similarities to native GLP-1 and activate the GLP-1 receptor at a supra-physiological level. ${ }^{1}$ Both agents can be used to manage the blood glucose with low risk of hypoglycemia by suppressing glucagon secretion and enhancing insulin secretion in a glucose-dependent manner. ${ }^{2}$ GLP-1RAs also suppress gastric emptying and satiety, resulting in the induction of weight reduction. ${ }^{2}$ Furthermore, GLP-1RAs were reported to reduce cardiovascular disease (CVD) events, ie, 3-point MACE (cardiovascular death, non-fatal myocardial infarction, non-fatal stroke), in several trials ${ }^{3-7}$ performed to verify their cardiovascular safety, as well as in a meta-analysis. ${ }^{8,9}$ In light of these evidences, the American Diabetes Association recommends that patients with type 2 diabetes (T2DM) and established atherosclerotic cardiovascular disease (ASCVD), indicators of high risk for ASCVD, or chronic kidney disease are considered for treatment with GLP-1RAs. ${ }^{10}$ However, the heterogeneity of the efficacy or safety for patients with T2DM among GLP-1RAs remains unclear. Exenatide, one of the GLP-1RAs, is a synthetic GLP-1RA derived from exendin-4. ${ }^{11}$ Treatment with exenatide $2 \mathrm{mg}$ once weekly (QW) as a long-acting formulation was shown to achieve a significant reduction in fasting plasma glucose. ${ }^{12}$ In this review, we outline the current evidence regarding the clinical efficacy and safety of exenatide QW in patients with T2DM, and discuss the current perspectives on the use of exenatide QW. 


\section{Search Strategy}

This mini-review performed a search using PubMed with the search terms "exenatide", "exenatide once weekly", "incretin", "GLP-1", "GLP-1 receptor agonist", "cardiovascular outcome", "renal outcome", and "type 2 diabetes". In addition, relevant articles were collected from the personal databases of the authors.

\section{Pharmacokinetics of Exenatide QW}

Exendin-4 was discovered in a search for biologically active peptides in the venom of the Gila monster. ${ }^{13}$ This reptilian protein shares about 50\% amino acid sequence identity with mammalian GLP-1 and is resistant to DPP-4-mediated degradation. Exenatide, lixisenatide and efpeglenatide have all been developed based on exendin-4. Although liraglutide, semaglutide, dulaglutide and albiglutide were based on human GLP-1, it is unclear whether there is any difference in the effects deriving from the structural basis of the GLP-1RAs used for management of T2DM. Exenatide QW was developed as a long-acting formulation with once-weekly administration and consists of microspheres composed of a biodegradable poly (lactide-co-glycolide) polymeric matrix containing the peptide exenatide. ${ }^{14}$ Exenatide is slowly released from the microspheres through diffusion and microsphere breakdown following once-weekly subcutaneous injection, ${ }^{15}$ and a steady-state plasma concentration is reached after 6-8 weeks of treatment.

\section{Clinical Efficacy of Exenatide QW}

The efficacy and safety of exenatide QW have been evaluated in the DURATION trials (The Diabetes Therapy Utilization: Researching Changes in A1C, Weight and Other Factors Through Intervention with Exenatide Once Weekly). ${ }^{16-25}$ The efficacy outcomes of exenatide QW in the DURATION trials are shown in Table 1. The DURATION trials (excluding DURATION 7 due to the presence of a titration phase with insulin glargine) reported reductions in fasting blood glucose (FBG), HbAlc and body weight from the baseline in the range of 32-46 mg/dl, 1.3-1.6\% and 2.0-3.7 kg, respectively, throughout 24-30 weeks of treatment with exenatide QW. DURATION 1 and 5 assessed the clinical effects of exenatide QW compared to exenatide twice daily (BID), and found that treatment with exenatide QW was superior to exenatide BID in reducing $\mathrm{HbAlc}$ and FBG. ${ }^{16,17,22}$ DURATION 2 and 4 reported treatment with exenatide QW was superior to sitagliptin for reductions in $\mathrm{HbA1c}, \mathrm{FBG}$ and body weight, and equivalent or superior to pioglitazone for reductions in HbA1c and FBG in addition to clear benefits for body weight. ${ }^{18,21}$ DURATION 4 also reported that treatment with exenatide QW was equivalent to metformin for reductions in HbA1c, FBG and body weight. ${ }^{21}$ DURATION 3 demonstrated the superiority of treatment with exenatide QW for reductions in HbAlc and body weight compared with insulin glargine, in spite of the inferiority of FBG. ${ }^{20}$ DURATION 7 showed reductions in FBG, HbAlc and body weight of $12 \mathrm{mg} / \mathrm{dl}, 1.0 \%$ and $1.0 \mathrm{~kg}$ from baseline, respectively, in patients treated with exenatide QW after an 8-week titration phase with insulin glargine. ${ }^{24}$ DURATION 8 reported that treatment with exenatide QW plus dapagliflozin was superior to either drug alone for reductions in $\mathrm{HbAlc}$, FBG and body weight, demonstrating an additive effect between these two agents. ${ }^{25}$ Both treatment with exenatide QW and treatment with daily liraglutide $1.8 \mathrm{mg}$ led to reductions in FBG, HbA1c and body weight in DURATION 6, and greater reductions were noted with liraglutide. ${ }^{23}$ Thus, exenatide QW has been reported to reduce body weight, but is not approved for treatment of obesity, while liraglutide ( $3.0 \mathrm{mg}$ once-daily) has been approved for treatment of obesity by the US Food and Drug Administration (FDA). In the DURATION trials, treatments for T2DM with exenatide QW showed the clinical efficacy though not as effective as liraglutide $1.8 \mathrm{mg}$, suggesting that exenatide QW is an option for the treatment of T2DM, but it might be used with lower priority compared to liraglutide.

\section{Safety Concerns}

The most common adverse events of GLP-1RAs are nausea, vomiting and diarrhea, and these adverse events associated with exenatide QW were also most frequently observed in the DURATION trials. ${ }^{12,26}$ Gastrointestinal adverse events caused by GLP-1RAs often occur within the first month of treatment and tend to diminish within a couple of weeks. ${ }^{27,28}$ In an integrated analysis of 4328 patients in eight trials including the DURATION1-6 studies to assess the safety and tolerability of exenatide $\mathrm{QW},{ }^{28}$ the percentage of patients experiencing nausea and vomiting was smaller among those administered exenatide QW (14.4\% and 6.7\%, respectively) than those treated with exenatide BID (29.9\% and $13.0 \%)$, 
Table I Efficacy Outcomes of Exenatide QW in the DURATION Trials

\begin{tabular}{|c|c|c|c|c|c|c|c|}
\hline \multirow[t]{2}{*}{ Study } & \multirow[t]{2}{*}{ Subjects } & \multirow[t]{2}{*}{ Background } & \multirow[t]{2}{*}{$\begin{array}{l}\text { Intervention and } \\
\text { Comparators }\end{array}$} & \multirow[t]{2}{*}{$\begin{array}{l}\text { Duration } \\
\text { (Weeks) }\end{array}$} & \multicolumn{3}{|c|}{$\begin{array}{l}\text { Endpoints, Changes from the Baselines } \\
\text { (Baseline Data) }\end{array}$} \\
\hline & & & & & FBG (mg/dl) & HbAlc (\%) & Weight (kg) \\
\hline \multirow{2}{*}{$\begin{array}{l}\text { DURATION-I }{ }^{16} \\
\text { Drucker et al, } 2008\end{array}$} & \multirow[t]{2}{*}{295} & \multirow{2}{*}{$\begin{array}{l}\text { Lifestyle modification and } \\
\text { oral antihyperglycemic } \\
\text { drugs, age } \geq 16 \text { years, } \mathrm{HbAlc} \\
7.1-11.0 \%, \mathrm{FBG} \leq 288 \mathrm{mg} / \mathrm{dl} \text {, } \\
\text { BMI } 25-45 \mathrm{~kg} / \mathrm{m}^{2}\end{array}$} & Exenatide QW & \multirow[t]{2}{*}{30} & $-4 \mid(\mid 73)$ & $-1.9(8.3)$ & $-3.7(102)$ \\
\hline & & & Exenatide BID & & $-25^{*}(166)$ & $-1.5 *(8.3)$ & $-3.6(102)$ \\
\hline \multirow{3}{*}{$\begin{array}{l}\text { DURATION-2 }{ }^{18} \\
\text { Bergenstal et al, } 2010\end{array}$} & \multirow[t]{3}{*}{491} & \multirow{3}{*}{$\begin{array}{l}\text { Metformin, age } \geq 18 \text { years, } \\
\text { HbAlc } 7.1-11.0 \% \text {, BMI } 25- \\
45 \mathrm{~kg} / \mathrm{m}^{2}\end{array}$} & Exenatide QW & \multirow[t]{3}{*}{26} & $-32(166)$ & $-1.5(8.6)$ & $-2.3(89)$ \\
\hline & & & Sitagliptin $100 \mathrm{mg} /$ day & & $-16 *(164)$ & $-0.9 *(8.5)$ & $-0.8 *(87)$ \\
\hline & & & Pioglitazone $45 \mathrm{mg} /$ day & & $-27(164)$ & $-1.2^{*}(8.5)$ & $+2.8 *(88)$ \\
\hline \multirow{2}{*}{$\begin{array}{l}\text { DURATION-3 }{ }^{20} \\
\text { Diamant et al, } 2010\end{array}$} & \multirow[t]{2}{*}{456} & \multirow{2}{*}{$\begin{array}{l}\text { Metformin and } / \text { or } \\
\text { sulphonylurea, age } \geq 18 \\
\text { years, HbAIc } 7.1-11.0 \% \text {, } \\
\text { BMI } 25-45 \mathrm{~kg} / \mathrm{m}^{2}\end{array}$} & Exenatide QW & \multirow[t]{2}{*}{26} & $-38(178)$ & $-1.5(8.3)$ & $-2.6(91.2)$ \\
\hline & & & Insulin glargine & & $-50 *(175)$ & $-1.3 *(8.3)$ & $+1.4^{*}(90.6)$ \\
\hline \multirow{4}{*}{$\begin{array}{l}\text { DURATION-4 } \\
\text { Russell-Jones et al, } 2012\end{array}$} & \multirow[t]{4}{*}{820} & \multirow{4}{*}{$\begin{array}{l}\text { Lifestyle modification, } \\
\mathrm{HbAlc} 7 . \mathrm{I}-\mathrm{II} .0 \% \text {, BMI } 23- \\
45 \mathrm{~kg} / \mathrm{m}^{2}\end{array}$} & Exenatide QW & \multirow[t]{4}{*}{26} & $-4 \mid(\mid 78)$ & $-1.5(8.5)$ & $-2.0(87.5)$ \\
\hline & & & Metformin 2000 mg/day & & $-36(180)$ & $-1.5(8.6)$ & $-2.0(85.9)$ \\
\hline & & & Pioglitazone $45 \mathrm{mg} /$ day & & $-47(176)$ & $-1.6(8.5)$ & $+1.5 *(86.1)$ \\
\hline & & & Sitagliptin $100 \mathrm{mg} /$ day & & $-20 *(175)$ & $-1.2 *(8.5)$ & $-0.8^{*}(88.7)$ \\
\hline \multirow{2}{*}{$\begin{array}{l}\text { DURATION }-5^{22} \\
\text { Blevins et al, } 2011\end{array}$} & \multirow[t]{2}{*}{252} & \multirow{2}{*}{$\begin{array}{l}\text { Lifestyle modification and } \\
\text { oral antihyperglycemic } \\
\text { drugs, age } \geq 18 \text { years, } \mathrm{HbAlc} \\
7.1-1 \mathrm{l} .0 \%, \mathrm{FBG} \leq 280 \mathrm{mg} / \mathrm{dl} \text {, } \\
\text { BMI } 25-45 \mathrm{~kg} / \mathrm{m}^{2}\end{array}$} & Exenatide QW & \multirow[t]{2}{*}{24} & $-35(173)$ & $-1.6(8.5)$ & $-2.3(97.0)$ \\
\hline & & & Exenatide BID & & $-12 *(168)$ & $-0.9 *(8.4)$ & $-1.4(94.3)$ \\
\hline \multirow{2}{*}{$\begin{array}{l}\text { DURATION }-6^{23} \\
\text { Buse et al, } 2013\end{array}$} & \multirow[t]{2}{*}{911} & \multirow{2}{*}{$\begin{array}{l}\text { Lifestyle modification and } \\
\text { oral antihyperglycemic } \\
\text { drugs, age } \geq 18 \text { years, HbAlc } \\
7.1-11.0 \%, \text { BMI } \leq 45 \mathrm{~kg} / \mathrm{m}^{2}\end{array}$} & Exenatide QW & \multirow[t]{2}{*}{26} & $-32(173)$ & $-1.3(8.5)$ & $-2.7(90.9)$ \\
\hline & & & Liraglutide $1.8 \mathrm{mg} /$ day & & $-40 *(176)$ & $-1.5 *(8.4)$ & $-3.6 *(91.1)$ \\
\hline \multirow[t]{2}{*}{$\begin{array}{l}\text { DURATION-7 }{ }^{24} \\
\text { Guja et al, } 2018\end{array}$} & \multirow[t]{2}{*}{464} & \multirow{2}{*}{$\begin{array}{l}\text { Lifestyle modification and } \\
\text { metformin and/or } \\
\text { sulphonylurea, age } \geq 18 \\
\text { years, HbAIc } 7.0-10.5 \% \text { at } \\
\text { randomization after an } \\
\text { 8-week titration phase }\end{array}$} & $\frac{\text { Exenatide } \mathrm{QW}+\text { insulin }}{\text { glargine } \pm \text { metformin }}$ & \multirow[t]{2}{*}{28} & $-12(148)$ & $-1.0(8.5)$ & $-1.0(94.2)$ \\
\hline & & & $\begin{array}{l}\text { Placebo + insulin glargine } \\
\pm \text { metformin }\end{array}$ & & $-2^{*}(144)$ & $-0.2 *(8.5)$ & $+0.5^{*}(94.1)$ \\
\hline DURATION $-8^{25}$ & 685 & Metformin, age $\geq 18$ years, & Exenatide QW & 28 & $-46(189)$ & $-1.6(9.3)$ & $-1.6(89.1)$ \\
\hline & & HbAlc $8.0-12.0 \%$ & Dapagliflozin $10 \mathrm{mg} /$ day & & $-49(188)$ & $-1.4(9.3)$ & $-2.2(90.9)$ \\
\hline & & & $\begin{array}{l}\text { Exenatide } \mathrm{QW}+ \\
\text { dapagliflozin } 10 \mathrm{mg} / \text { day }\end{array}$ & & $-66 *(195)$ & $-2.0 *(9.3)$ & $-3.6 *(92.1)$ \\
\hline
\end{tabular}

Notes: ${ }^{p}<0.05$, versus comparators. The underlines in Table I show comparators.

Abbreviations: T2DM, type 2 diabetes; QW, once-weekly formulation; BID, twice-daily formulation; FBG, fasting blood glucose.

but they were larger among patients given exenatide QW compared to patients treated without GLP-1RAs (4.9\% and $2.5 \%)$. In this analysis, diarrhea was experienced by a higher percentage of patients administered exenatide QW (10.5\%) than patients administered exenatide BID (8.4\%) or comparators without GLP-1RAs (7.4\%). DURATION 6 reported that nausea, vomiting, and diarrhea occurred in a smaller percentage of patients treated with exenatide QW $(9.3 \%, 3.7 \%$, and $6.1 \%$, respectively) than patients treated with liraglutide $1.8 \mathrm{mg} /$ day $(20.7 \%, 10.7 \%$, and $13.1 \%) .{ }^{23,28}$ On the other hand, adverse events related to injection-site reactions - which were mainly induration, nodule and pruritus - were observed in 
a greater percentage of patients treated with exenatide QW (16-20\%) compared to those administered exenatide BID and liraglutide. However, cases of discontinuation due to adverse events related to injection-site reactions seemed to be limited, occurring in only $0.2-0.8 \%$ of patients treated with exenatide $\mathrm{QW} .{ }^{28}$ Adverse events related to pancreatitis, renal failure, gallbladder disease, and thyroid cancer were rarely reported in patients treated with exenatide QW. ${ }^{28}$ Thus, treatments with exenatide QW were shown to be clinically safe, and gastrointestinal adverse events such as nausea and vomiting were less frequent in the treatment with exenatide QW compared to those with exenatide BID or liraglutide.

\section{Effects of Switching from Exenatide BID to Exenatide QW}

According to the pharmacokinetic/pharmacodynamic profiles, GLP-1RAs are categorized as either short-acting compounds, which predominantly suppress postprandial glucose levels by delaying gastric emptying, or as long-acting compounds, which predominantly reduce fasting plasma glucose levels by stimulating insulin secretion and suppressing glucagon secretion. ${ }^{1}$ Exenatide has two formulations: a short-acting BID formulation and a long-acting QW formulation. The effects of switching from exenatide BID to exenatide QW were elucidated in a previous study by our group ${ }^{29}$ as well as in studies by other authors, ${ }^{30,31}$ switching from short- to long-acting formulations induces reductions in HbAlc and FBG, and increases of postprandial hyperglycemia. Weights after switching were reported to be unchanged in two of the studies. $^{29,31}$ We reported an improvement in treatment satisfaction in patients assessed by the Diabetes Treatment Satisfaction Questionnaire, and a reduced incidence of hypoglycemia after switching. ${ }^{29}$ We also recently reported that despite the increase in postprandial glucose associated with accelerated gastric emptying after switching from exenatide BID to QW, change in glycemic variability assessed by continuous glucose monitoring was modest, and no significant deterioration in vascular endothelial function was observed after switching. ${ }^{32}$ These findings suggest that treatment with exenatide QW is more useful than treatment with exenatide BID in clinical practice.

\section{Cardiovascular Outcome}

The Exenatide Study of Cardiovascular Event Lowering (EXSCEL) was a randomized, double-blind and placebo-controlled trial to investigate the cardiovascular effects of treatment with exenatide QW in patients with T2DM. ${ }^{33}$ This trial enrolled 14,752 patients, 10,782 (73\%) of whom had CVD, and followed them for a median of 3.2 years. The primary outcome was evaluated as a composite endpoint of 3-point MACE, and occurred in 839 patients (11.4\%) with exenatide QW compared to 905 patients $(12.2 \%)$ in the placebo group. As a result, treatment with exenatide QW was shown to be noninferior to placebo $(\mathrm{P}<0.001)$, but failed to reduce the 3-point MACE composite compared with placebo (hazard ratio (HR) 0.91 ; 95\% CI 0.83-1.00, $\mathrm{P}=0.06$ for superiority). While some GLP-1RAs such as lixisenatide and oral semaglutide also achieved no significant reduction in CVD outcomes, ${ }^{34,35}$ other GLP-1RAs such as liraglutide, semaglutide, albiglutide and dulaglutide achieved $13 \%$ (HR $0.87,95 \%$ CI $0.78-0.97),{ }^{3} 26 \%$ (HR 0.74, 95\% CI 0.58-0.95), ${ }^{4} 22 \%$ (HR 0.78, 95\% CI 0.68-0.90) ${ }^{5}$ and $12 \%$ (HR 0.88, 95\% CI 0.79-0.99) reductions in the 3-point MACE composite in their respective trials. ${ }^{36}$ Efpeglenatide, which consists of a modified exendin-4 molecule conjugated to an IgG4 Fc fragment, ${ }^{37}$ is one of the long-acting GLP-1RAs. In the recent AMPLITUDE-O trial, treatment with efpeglenatide 4 or $6 \mathrm{mg}$ QW was reported to reduce the incidence of MACE (HR $0.73,95 \%$ CI $0.58-0.92$ ) and renal endpoint, as assessed by the composite of macroalbuminuria or a decrease in kidney function (HR $0.68,95 \%$ CI $0.57-0.79$ ), compared to placebo. ${ }^{7}$ Of note, the reduction in MACE in this trial was observed regardless of using metformin or SGLT2 inhibitor at baseline, while previous trials included limited persons treated with an SGLT2 inhibitor. A recent meta-analysis of AMPLITUDE-O and seven other trials, which was designed to evaluate the cardiovascular benefits and risks of GLP-1RAs, reported that human and exendin-4-based agents have similar benefits for MACE regardless of the structural basis of the GLP1RAs. ${ }^{9}$ Treatment with efpeglenatide $4 \mathrm{mg} \mathrm{QW}$ was shown to reduce $\mathrm{HbA1c}$ and body weight comparable to those by liraglutide $1.8 \mathrm{mg} /$ day. ${ }^{38}$ In the head-to-head trial of exenatide QW and semaglutide 1.0 mg QW (SUSTAIN 3), treatment with semaglutide was superior to exenatide QW in reducing HbAlc ( $-1.5 \%$ vs $-0.9 \%)$, FBG $(-50 \mathrm{mg} / \mathrm{dl} \mathrm{vs}-36 \mathrm{mg} / \mathrm{dl})$ and body weight $(-5.6 \mathrm{~kg}$ vs $-1.9 \mathrm{~kg})$ after 56 weeks of treatment. ${ }^{39}$ Moreover, a retrospective cohort study employing an electronic medical record database reported that treatment with exenatide QW achieved an $\mathrm{HbAlc}$ reduction equivalent to that by dulaglutide $1.5 \mathrm{mg}$, but a smaller reduction in body weight compared to dulaglutide. ${ }^{40}$ Although the reasons for the heterogeneity of effects on CVD among GLP-1RAs remain unclear, ${ }^{8}$ the greater efficacy for glycemic control or body weight achieved by liraglutide (DURATION 6) ${ }^{23}$ semaglutide (SUSTAIN 3) ${ }^{4}$ and dulaglutide ${ }^{40}$ compared to exenatide QW might affect the incidence of 
CVD. Indeed, a meta-analysis of 12 trials evaluating the risk of MACE for treatment with the newer glucose-lowering drugs, including GLP-1RAs, suggested a relation between the reduction of HbAlc and the lower HR of MACE. ${ }^{41}$

\section{Renal Outcome}

GLP-1RAs reduced the incidence of composite renal outcomes in several trials. ${ }^{3,4,7,42}$ However, this effect was driven mainly by the reduction in albuminuria. It remains unclear whether GLP-1RAs are able to improve renal outcomes such as worsening of kidney function. ${ }^{9}$ A significant reduction in renal outcomes, as assessed by the composite of $40 \%$ estimated glomerular filtration rate (eGFR) decline, renal death, renal replacement and new macroalbuminuria, was reported with exenatide QW in an adjusted analysis (HR 0.85, 95\% CI 0.73-0.98). ${ }^{43}$ Finally, in the post hoc EXSCEL analysis, treatments with exenatide QW improved both the urinary albumin excretion and eGFR decline in subjects with elevated baseline urinary albumin compared to placebo, suggesting that exenatide QW reduces urinary albumin and improves the eGFR slope specifically in subjects with an elevated baseline urinary albumin: creatinine ratio. ${ }^{44}$

\section{Possible Mechanisms of Cardiovascular Outcome}

The separation of the Kaplan-Meier curves for CVD events among treatments in the trials of GLP-1RAs appeared to occur gradually compared to the trials of sodium-glucose cotransporter 2 (SGLT2) inhibitors such as EMPA-REG OUTCOME trial. ${ }^{45}$ These results suggest that GLP-1RAs achieve a reduction in CVD events through their antiatherosclerotic effects, in contrast to the hemodynamic effects associated with SGLT2 inhibitors. ${ }^{46}$ A recent study demonstrated no significant difference in carotid plaque progression for 18 months between treatment with exenatide QW and placebo, ${ }^{47}$ suggesting that longer duration of treatment with exenatide QW may be needed to exert antiatherosclerotic effects. Other possible mechanisms of cardiorenal protection by GLP-1RAs, such as anti-inflammatory, anti-fibrotic, anti-oxidative or vasodilatory effects, have been noted in other studies. ${ }^{36,48,49}$

\section{Current Perspectives}

Treatments with exenatide QW showed clinical efficacy and safety and achieved borderline reductions in CVD for patients with T2DM in the above trials. The five- or seven-year follow-up studies in the DURATION-1 trial reported sustained improvements in glycemic control and no unexpected safety findings by treatment with exenatide QW, ${ }^{50,51}$ demonstrating the long-term usefulness of exenatide QW in clinical practice. In addition, once-weekly GLP-1RAs, including exenatide $\mathrm{QW}$, are more convenient, and are therefore preferred by patients and more likely to encourage treatment adherence compared to agents which need daily injection. ${ }^{52-54}$ However, taking the results from the trials of other GLP-1RAs together, exenatide QW might be considered less preferable than other GLP1-RAs for the management of T2DM, especially for patients with high CV risk. Table 2 shows the outcomes of trials using GLP-1RAs based on exendin-4 (exenatide QW, lixisenatide and efpeglenatide). The results indicate that, among the GLP1-RAs based on exendin-4, efpeglenatide may be the first choice if it becomes available. On the other hand, from the perspective of price and utility, treatment with exenatide QW may be continued in patients with optimal glycemic control without adverse events. Additionally, the FDA approved exenatide QW for pediatric use in children aged 10 to 17 years with T2DM based on the positive Phase 3 data. ${ }^{55}$ Exenatide QW was also reported to cause weight loss in subjects aged 10 to 18 years with obesity, ${ }^{56}$ and thus this agent is expected to be effective for the management of glycemia and body weight, and to provide a new option as the first once-weekly injectable GLP-1RA approved for children and adolescents. Furthermore, exenatide QW as well as BID was reported to exhibit positive effects on motor scores in patients with Parkinson's disease compared to the placebo. ${ }^{57,58}$ Although it is unclear how to improve motor symptoms for patients with Parkinson's disease, exenatide is expected to have neuroprotective effects in this disease and other neurodegenerative disorders. ${ }^{59}$ Thus, further verification of the effects of exenatide QW on children and adults with T2DM or obesity, as well as patients with neurodegenerative disorders, will be needed in the future.

\section{Conclusion}

This review summarized the current evidence of the clinical efficacy and safety of exenatide QW, and discussed future perspectives on the use of exenatide QW. GLP-1RAs are becoming the mainstay of therapeutic strategies for T2DM 
Table 2 Summary of Cardiovascular Outcome Trials with Exendin-Based Glucagon-Like Peptide-I Receptor Agonists

\begin{tabular}{|c|c|c|c|}
\hline & ELIXA $^{34,60}$ & EXSCEL $^{33,43}$ & AMPLITUDE-O ${ }^{7}$ \\
\hline Drug & Lixisenatide & Exenatide QW & Efpeglenatide \\
\hline Dose & 10 or $20 \mu \mathrm{g}$, once daily & $2 \mathrm{mg}$, once weekly & 4 or $6 \mathrm{mg}$, once weekly \\
\hline Number of participants & 6068 & 14,752 & 4076 \\
\hline Median follow-up period, years & 2.1 & 3.2 & 2.1 \\
\hline Mean age, years & 60.3 & 62.0 & 64.5 \\
\hline Sex (male), \% & 69 & 62 & 67 \\
\hline Mean $\mathrm{HbAlc}$ at baseline, $\%$ & 7.7 & 8.1 & 8.9 \\
\hline Mean BMI at baseline, $\mathrm{kg} / \mathrm{m}^{2}$ & 30.1 & 32.7 & 32.7 \\
\hline Established cardiovascular disease, \% & 100 & 73 & 90 \\
\hline 3-point MACE & HR I.02 (0.89-I.I7) $)^{\mathrm{a}}$ & HR 0.91 (0.83-1.00) & HR $0.73(0.58-0.92)$ \\
\hline All-cause mortality & HR $0.94(0.78-I .13)$ & HR $0.86(0.77-0.97)$ & HR $0.78(0.58-I .06)$ \\
\hline Cardiovascular death & HR $0.98(0.78-I .22)$ & HR $0.88(0.76-1.02)$ & HR $0.72(0.50-1.03)$ \\
\hline Fatal or non-fatal myocardial infarction & HR I.03 (0.87-I.22) & HR $0.97(0.85-1.10)$ & HR $0.75(0.54-1.05)$ \\
\hline Fatal or non-fatal stroke & HR I.I2 (0.79-I.58) & HR 0.85 (0.70-1.03) & HR 0.74 (0.47-I.I7) \\
\hline Renal outcome ${ }^{b}$ & HR $0.84(0.68-1.02)$ & HR $0.88(0.76-1.01)$ & HR $0.68(0.57-0.79)$ \\
\hline Worsening of kidney function & HR I.I6 (0.74-I.83) & HR 0.88 (0.74-I.05) & HR $0.35(0.10-1.27)$ \\
\hline
\end{tabular}

Notes: ${ }^{\text {a }}$-point MACE (cardiovascular death, non-fatal myocardial infarction, non-fatal stroke, hospitalization for unstable angina) was reported in ELIXA. ${ }^{\mathrm{R}}$ Renal outcome comprised composite kidney outcome including new-onset macroalbuminuria.

Abbreviations: 3-point MACE, major adverse cardiovascular events (cardiovascular death, non-fatal myocardial infarction, non-fatal stroke); HR, hazard ratio.

based on the positive evidence of CV benefits. However, within the class of GLP1-RAs, exenatide QW may not be a first choice for adult patients with T2DM, and especially for patients at high CV risk: despite its long-term efficacy and safety, exenatide QW has not been clearly shown to have CV benefits.

\section{Acknowledgments}

The authors were supported by grants from the Ministry of Education, Culture, Sports, Science and Technology (Grant Nos. 20K17542 and 21K08535).

\section{Disclosure}

J.I. reports no conflicts of interest in this work. Y.S. received honoraria from Sumitomo Dainippon Pharma Co.

\section{References}

1. Meier JJ. GLP-1 receptor agonists for individualized treatment of type 2 diabetes mellitus. Nat Rev Endocrinol. 2012;8(12):728-742. doi:10.1038/ nrendo.2012.140

2. Saisho Y. Incretin-based therapy and pancreatitis: accumulating evidence and unresolved questions. Ann Transl Med. 2018;6(7):131. doi:10.21037/ atm.2018.02.24

3. Marso SP, Daniels GH, Brown-Frandsen K, et al. Liraglutide and cardiovascular outcomes in type 2 diabetes. N Engl J Med. 2016;375(4):311-322. doi:10.1056/NEJMoa1603827

4. Marso SP, Bain SC, Consoli A, et al. Semaglutide and cardiovascular outcomes in patients with type 2 diabetes. $N$ Engl J Med. 2016;375 (19):1834-1844. doi:10.1056/NEJMoa1607141

5. Hernandez AF, Green JB, Janmohamed S, et al. Albiglutide and cardiovascular outcomes in patients with type 2 diabetes and cardiovascular disease (Harmony Outcomes): a double-blind, randomised placebo-controlled trial. Lancet. 2018;392(10157):1519-1529. doi:10.1016/S0140-6736(18) 32261-X 
6. Gerstein HC, Colhoun HM, Dagenais GR, et al. Dulaglutide and cardiovascular outcomes in type 2 diabetes (REWIND): a double-blind, randomised placebo-controlled trial. Lancet. 2019;394(10193):121-130. doi:10.1016/S0140-6736(19)31149-3

7. Gerstein HC, Sattar N, Rosenstock J, et al. Cardiovascular and renal outcomes with efpeglenatide in type 2 diabetes. $N$ Engl J Med. 2021;385 (10):896-907. doi:10.1056/NEJMoa2108269

8. Bethel MA, Patel RA, Merrill P, et al. Cardiovascular outcomes with glucagon-like peptide-1 receptor agonists in patients with type 2 diabetes: a meta-analysis. Lancet Diabetes Endocrinol. 2018;6(2):105-113. doi:10.1016/S2213-8587(17)30412-6

9. Sattar N, Lee MMY, Kristensen SL, et al. Cardiovascular, mortality, and kidney outcomes with GLP-1 receptor agonists in patients with type 2 diabetes: a systematic review and meta-analysis of randomised trials. Lancet Diabetes Endocrinol. 2021;9(10):653-662. doi:10.1016/S22138587(21)00203-5

10. Association AD. 9. Pharmacologic approaches to glycemic treatment: standards of medical care in diabetes-2021. Diabetes Care. 2021;44(Suppl1): S111-s24.

11. Knop FK, Brønden A, Vilsbøll T. Exenatide: pharmacokinetics, clinical use, and future directions. Expert Opin Pharmacother. 2017;18 (6):555-571. doi:10.1080/14656566.2017.1282463

12. Heimbürger SM, Brønden A, Johansen NJ, et al. The efficacy and safety of exenatide once weekly in patients with type 2 diabetes. Expert Opin Pharmacother. 2019;20(5):501-510. doi:10.1080/14656566.2019.1571040

13. Eng J, Kleinman WA, Singh L, et al. Isolation and characterization of exendin-4, an exendin-3 analogue, from Heloderma suspectum venom. Further evidence for an exendin receptor on dispersed acini from Guinea pig pancreas. J Biol Chem. 1992;267(11):7402-7405. doi:10.1016/S00219258(18)42531-8

14. DeYoung MB, MacConell L, Sarin V, et al. Encapsulation of exenatide in poly-(D,L-lactide-co-glycolide) microspheres produced an investigational long-acting once-weekly formulation for type 2 diabetes. Diabetes Technol Ther. 2011;13(11):1145-1154. doi:10.1089/dia.2011.0050

15. Kim D, MacConell L, Zhuang D, et al. Effects of once-weekly dosing of a long-acting release formulation of exenatide on glucose control and body weight in subjects with type 2 diabetes. Diabetes Care. 2007;30(6):1487-1493. doi:10.2337/dc06-2375

16. Drucker DJ, Buse JB, Taylor K, et al. Exenatide once weekly versus twice daily for the treatment of type 2 diabetes: a randomised, open-label, non-inferiority study. Lancet. 2008;372(9645):1240-1250. doi:10.1016/S0140-6736(08)61206-4

17. Buse JB, Drucker DJ, Taylor KL, et al. DURATION-1: exenatide once weekly produces sustained glycemic control and weight loss over 52 weeks. Diabetes Care. 2010;33(6):1255-1261. doi:10.2337/dc09-1914

18. Bergenstal RM, Wysham C, Macconell L, et al. Efficacy and safety of exenatide once weekly versus sitagliptin or pioglitazone as an adjunct to metformin for treatment of type 2 diabetes (DURATION-2): a randomised trial. Lancet. 2010;376(9739):431-439. doi:10.1016/S0140-6736(10) 60590-9

19. Wysham C, Bergenstal R, Malloy J, et al. DURATION-2: efficacy and safety of switching from maximum daily sitagliptin or pioglitazone to once-weekly exenatide. Diabet Med. 2011;28(6):705-714. doi:10.1111/j.1464-5491.2011.03301.x

20. Diamant M, Van Gaal L, Stranks S, et al. Once weekly exenatide compared with insulin glargine titrated to target in patients with type 2 diabetes (DURATION-3): an open-label randomised trial. Lancet. 2010;375(9733):2234-2243. doi:10.1016/S0140-6736(10)60406-0

21. Russell-Jones D, Cuddihy RM, Hanefeld M, et al. Efficacy and safety of exenatide once weekly versus metformin, pioglitazone, and sitagliptin used as monotherapy in drug-naive patients with type 2 diabetes (DURATION-4): a 26-week double-blind study. Diabetes Care. 2012;35(2):252-258. doi: $10.2337 / \mathrm{dc} 11-1107$

22. Blevins T, Pullman J, Malloy J, et al. DURATION-5: exenatide once weekly resulted in greater improvements in glycemic control compared with exenatide twice daily in patients with type 2 diabetes. J Clin Endocrinol Metab. 2011;96(5):1301-1310. doi:10.1210/jc.2010-2081

23. Buse JB, Nauck M, Forst T, et al. Exenatide once weekly versus liraglutide once daily in patients with type 2 diabetes (DURATION-6): a randomised, open-label study. Lancet. 2013;381(9861):117-124. doi:10.1016/S0140-6736(12)61267-7

24. Guja C, Frías JP, Somogyi A, et al. Effect of exenatide QW or placebo, both added to titrated insulin glargine, in uncontrolled type 2 diabetes: the DURATION-7 randomized study. Diabetes Obes Metab. 2018;20(7):1602-1614. doi:10.1111/dom.13266

25. Frías JP, Guja C, Hardy E, et al. Exenatide once weekly plus dapagliflozin once daily versus exenatide or dapagliflozin alone in patients with type 2 diabetes inadequately controlled with metformin monotherapy (DURATION-8): a 28 week, multicentre, double-blind, phase 3, randomised controlled trial. Lancet Diabetes Endocrinol. 2016;4(12):1004-1016. doi:10.1016/S2213-8587(16)30267-4

26. Genovese S, Mannucci E, Ceriello A. A review of the long-term efficacy, tolerability, and safety of exenatide once weekly for type 2 diabetes. $A d v$ Ther. 2017;34(8):1791-1814. doi:10.1007/s12325-017-0499-6

27. Nauck M. Incretin therapies: highlighting common features and differences in the modes of action of glucagon-like peptide-1 receptor agonists and dipeptidyl peptidase-4 inhibitors. Diabetes Obes Metab. 2016;18(3):203-216. doi:10.1111/dom.12591

28. MacConell L, Gurney K, Malloy J, et al. Safety and tolerability of exenatide once weekly in patients with type 2 diabetes: an integrated analysis of 4328 patients. Diabetes Metab Syndr Obes. 2015;8:241-253. doi:10.2147/DMSO.S77290

29. Watanabe Y, Saisho Y, Inaishi J, et al. Efficacy and safety of once-weekly exenatide after switching from twice-daily exenatide in patients with type 2 diabetes. J Diabetes Investig. 2020;11(2):382-388. doi:10.1111/jdi.13146

30. Ji L, Onishi Y, Ahn CW, et al. Efficacy and safety of exenatide once-weekly vs exenatide twice-daily in Asian patients with type 2 diabetes mellitus. $J$ Diabetes Investig. 2013;4(1):53-61. doi:10.1111/j.2040-1124.2012.00238.x

31. Onishi Y, Koshiyama H, Imaoka T, et al. Safety of exenatide once weekly for 52 weeks in Japanese patients with type 2 diabetes mellitus. $J$ Diabetes Investig. 2013;4(2):182-189. doi:10.1111/jdi.12000

32. Inaishi J, Saisho Y, Watanabe Y, et al. Changes in glycemic variability, gastric emptying and vascular endothelial function after switching from twice-daily to once-weekly exenatide in patients with type 2 diabetes: a subpopulation analysis of the twin-exenatide study. BMC Endocr Disord. 2022;22(1):20. doi:10.1186/s12902-022-00932-9

33. Holman RR, Bethel MA, Mentz RJ, et al. Effects of once-weekly exenatide on cardiovascular outcomes in type 2 diabetes. N Engl J Med. 2017;377 (13):1228-1239. doi:10.1056/NEJMoa1612917

34. Pfeffer MA, Claggett B, Diaz R, et al. Lixisenatide in patients with type 2 diabetes and acute coronary syndrome. $N$ Engl J Med. 2015;373 (23):2247-2257. doi:10.1056/NEJMoa1509225

35. Husain M, Birkenfeld AL, Donsmark M, et al. Oral semaglutide and cardiovascular outcomes in patients with type 2 diabetes. $N$ Engl $J$ Med. 2019;381(9):841-851. doi:10.1056/NEJMoa1901118 
36. Nagahisa T, Saisho Y. Cardiorenal protection: potential of SGLT2 inhibitors and GLP-1 receptor agonists in the treatment of type 2 diabetes. Diabetes Ther. 2019;10(5):1733-1752. doi:10.1007/s13300-019-00680-5

37. Sharma D, Verma S, Vaidya S, et al. Recent updates on GLP-1 agonists: current advancements \& challenges. Biomed Pharmacother. 2018;108:952-962. doi:10.1016/j.biopha.2018.08.088

38. Rosenstock J, Sorli CH, Trautmann ME, et al. Once-weekly efpeglenatide dose-range effects on glycemic control and body weight in patients with type 2 diabetes on metformin or drug naive, referenced to liraglutide. Diabetes Care. 2019;42(9):1733-1741. doi:10.2337/ dc18-2648

39. Ahmann AJ, Capehorn M, Charpentier G, et al. Efficacy and safety of once-weekly semaglutide versus exenatide ER in subjects with type 2 diabetes (SUSTAIN 3): a 56-week, open-label, randomized clinical trial. Diabetes Care. 2018;41(2):258-266. doi:10.2337/dc17-0417

40. Unni S, Wittbrodt E, Ma J, et al. Comparative effectiveness of once-weekly glucagon-like peptide-1 receptor agonists with regard to 6-month glycaemic control and weight outcomes in patients with type 2 diabetes. Diabetes Obes Metab. 2018;20(2):468-473. doi:10.1111/dom.13107

41. Giugliano D, Chiodini P, Maiorino MI, et al. Cardiovascular outcome trials and major cardiovascular events: does glucose matter? A systematic review with meta-analysis. $J$ Endocrinol Invest. 2019;42(10):1165-1169. doi:10.1007/s40618-019-01047-0

42. Gerstein HC, Colhoun HM, Dagenais GR, et al. Dulaglutide and renal outcomes in type 2 diabetes: an exploratory analysis of the REWIND randomised, placebo-controlled trial. Lancet. 2019;394(10193):131-138. doi:10.1016/S0140-6736(19)31150-X

43. Bethel MA, Mentz RJ, Merrill P, et al. Renal outcomes in the EXenatide Study of Cardiovascular Event Lowering (EXSCEL). Diabetes. 2018;67 (Supp11):522-P. doi:10.2337/db18-522-P

44. van der Aart-van der Beek AB, Clegg LE, Penland RC, et al. Effect of once-weekly exenatide on estimated glomerular filtration rate slope depends on baseline renal risk: a post hoc analysis of the EXSCEL trial. Diabetes Obes Metab. 2020;22(12):2493-2498. doi:10.1111/dom.14175

45. Zinman B, Wanner C, Lachin JM, et al. Empagliflozin, cardiovascular outcomes, and mortality in type 2 diabetes. $N$ Engl J Med. 2015;373 (22):2117-2128. doi:10.1056/NEJMoa1504720

46. Saisho Y. SGLT2 inhibitors: the star in the treatment of type 2 diabetes? Diseases. 2020;8(2):14. doi:10.3390/diseases 8020014

47. Koska J, Migrino RQ, Chan KC, et al. The effect of exenatide once weekly on carotid atherosclerosis in individuals with type 2 diabetes: an 18-month randomized placebo-controlled study. Diabetes Care. 2021;44(6):1385-1392. doi:10.2337/dc20-2014

48. Nauck MA, Quast DR. Cardiovascular safety and benefits of semaglutide in patients with type 2 diabetes: findings from SUSTAIN 6 and PIONEER 6. Front Endocrinol (Lausanne). 2021;12:645566. doi:10.3389/fendo.2021.645566

49. Alicic RZ, Cox EJ, Neumiller JJ, et al. Incretin drugs in diabetic kidney disease: biological mechanisms and clinical evidence. Nat Rev Nephrol. 2021;17(4):227-244. doi:10.1038/s41581-020-00367-2

50. Wysham CH, MacConell LA, Maggs DG, et al. Five-year efficacy and safety data of exenatide once weekly: long-term results from the DURATION-1 randomized clinical trial. Mayo Clin Proc. 2015;90(3):356-365. doi:10.1016/j.mayocp.2015.01.008

51. Philis-Tsimikas A, Wysham CH, Hardy E, et al. Efficacy and tolerability of exenatide once weekly over 7 years in patients with type 2 diabetes: an open-label extension of the DURATION-1 study. J Diabetes Complications. 2019;33(3):223-230. doi:10.1016/j.jdiacomp.2018.11.012

52. Qin L, Chen S, Flood E, et al. Glucagon-like Peptide-1 receptor agonist treatment attributes important to injection-naïve patients with type 2 diabetes mellitus: a Multinational Preference Study. Diabetes Ther. 2017;8(2):321-334. doi:10.1007/s13300-017-0230-2

53. Qiao Q, Ouwens MJ, Grandy S, et al. Adherence to GLP-1 receptor agonist therapy administered by once-daily or once-weekly injection in patients with type 2 diabetes in Germany. Diabetes Metab Syndr Obes. 2016;9:201-205. doi:10.2147/DMSO.S99732

54. Hauber AB, Nguyen H, Posner J, et al. A discrete-choice experiment to quantify patient preferences for frequency of glucagon-like peptide-1 receptor agonist injections in the treatment of type 2 diabetes. Curr Med Res Opin. 2016;32(2):251-262. doi:10.1185/03007995.2015.1117433

55. Healio. FDA approves once-weekly exenatide for children with type 2 diabetes. Available from: https://www.healio.com/news/Endocrinology/ 20210723/fda-approves-onceweekly-exenatide-for-children-with-type-2-diabetes. Accessed September 2, 2021.

56. Weghuber D, Forslund A, Ahlström H, et al. A 6-month randomized, double-blind, placebo-controlled trial of weekly exenatide in adolescents with obesity. Pediatr Obes. 2020;15(7):e12624. doi:10.1111/ijpo.12624

57. Athauda D, Maclagan K, Skene SS, et al. Exenatide once weekly versus placebo in Parkinson's disease: a randomised, double-blind, placebocontrolled trial. Lancet. 2017;390(10103):1664-1675. doi:10.1016/S0140-6736(17)31585-4

58. Mulvaney CA, Duarte GS, Handley J, et al. GLP-1 receptor agonists for Parkinson's disease. Cochrane Database Syst Rev. 2020;7(7):Cd012990. doi:10.1002/14651858.CD012990.pub2

59. Aviles-Olmos I, Dickson J, Kefalopoulou Z, et al. Exenatide and the treatment of patients with Parkinson's disease. J Clin Invest. 2013;123 (6):2730-2736. doi:10.1172/JCI68295

60. Muskiet MHA, Tonneijck L, Huang Y, et al. Lixisenatide and renal outcomes in patients with type 2 diabetes and acute coronary syndrome: an exploratory analysis of the ELIXA randomised, placebo-controlled trial. Lancet Diabetes Endocrinol. 2018;6(11):859-869. doi:10.1016/S2213$8587(18) 30268-7$

Clinical Pharmacology: Advances and Applications

Dovepress

\section{Publish your work in this journal}

Clinical Pharmacology: Advances and Applications is an international, peer-reviewed, open access journal publishing original research, reports, reviews and commentaries on all areas of drug experience in humans. The manuscript management system is completely online and includes a very quick and fair peer-review system, which is all easy to use. Visit http://www.dovepress.com/testimonials.php to read real quotes from published authors.

Submit your manuscript here: https://www.dovepress.com/clinical-pharmacology-advances-and-applications-journal 